\title{
Boundary values of holomorphic functions and some spectral problems for unitary repesentations
}

\author{
Yurii A. Neretin*
}

September 11, 2018

\author{
Moscow State Institute of Electronics and Mathematics, Bolshoi Triohsvy- \\ atitelskii, per. 3/12, Moscow-109028, Russia \\ Max-Planck-Institut für Mathematik, Gottfried-Claren-Str. 26, 53225 Bonn, \\ Germany \\ neretin mpim-bonn.mpg.de, neretin main.mccme.rssi.ru
}

Consider a (not necessarily irreducible) unitary representation of a semisimple Lie group $G$ in a Hilbert space $H$ (for instance we can consider an action of the group $G$ in some function space on some homogeneous space, a tensor product of irreducible unitary representations, a restriction of an irreducible unitary representation to a subgroup etc.). There arises a natural problem: to decompose our representation into irreducible representations, in other words, to find the spectrum of the representation. Problems of this type were widely investigated for the last 50 years (since [Kre]) and by now many such spectral problems have been solved completely or partially.

In several simple cases the spectra are continuous and more or less 'uniform'. But sometimes such spectra contain strange discrete increments which means that there exist minimal $G$-invariant subspaces in the Hilbert space $H$. First examples of discrete increments were observed in [Nai], [Puk], [H-Ch], [Mol1] (1961 - 1966) and now there exists a large literature devoted to discrete spectra, see [Boy], [Ism], [Mol3-4], [Str], [Far], [Pat], [F-J1], [F-J2], [Sch], [Kob1-2], [RSW], [Tsu], [How], [Ada], [Li], [BO] etc.

Quite often 'discrete increments' are very singular ('exotic') representations and it is very difficult to construct such unitary representations in some other way. Even Harish-Chandra's discrete series still cannot be considered handy objects. The remarkable paper [F-J1] (1980, see also [F-J2]) contained a serious generalization of Harish-Chandra's discrete series and this paper started a systematic search of 'exotic' unitary representations in discrete spectra.

*supported by RFRF (grant 95-01-00814) and Russian program of support of scientific schools (grant 96-01-96249) 
In [Ner1] (1986), [Ols3],[Ols4], [NO], [Ner2] it was observed that very often discrete increments to spectra are related to some functional analytic phenomena, namely to so-called 'trace theorems' (i.e theorems about restrictions of discontinuous functions to submanifolds). On various analytic trace theorems see [RS], IX.3,IX.9, [ESh], 2.3, [Beu], [Bar],5.12, [NR], [Rud], [Vla], [VS], [Ner2].

This paper is some kind of addendum to papers [NO], [Ner2] of Olshanskii and myself. Its purpose is to discuss some open problems related to applications of trace theorems in noncommutative harmonic analysis.

I am grateful to G. I. Olshanskii for our collaboration. I also thank V. F. Molchanov, H. Schlichtkrull, G. Zuckerman, B. Ørsted, M. Flensted-Jensen, T. Kobayshi, A. G. Sergeev, G. Ólafsson, B. Kostant, A. Dvorsky, R. S. Ismagilov, R. Howe, V. M. Gichev, V. V. Lebedev for discussions, comments and references. These notes are mainly based on my lectures [Ner5] delivered at the school 'Analysis on homogeneous spaces' (Tambov, august 1996) and I also thank the organizers of that school.

\section{Tensor product of two representations of $S L(2, \mathbb{R})$ of the complementary series}

1.1. Complementary series. Consider the circle $\mathbb{T}^{1}$ with the coordinates $z=e^{i \phi}$ (where $|z|=1, \phi \in[0,2 \pi]$ ). Let $-1<s<1, s \neq 0$. Further consider the scalar product

$$
<f_{1}, f_{2}>=\int_{0}^{2 \pi} \int_{0}^{2 \pi} \frac{f_{1}\left(\phi_{1}\right) \overline{f_{2}\left(\phi_{2}\right)} d \phi_{1} d \phi_{2}}{\left|\sin \left(\phi_{1}-\phi_{2}\right) / 2\right|^{(1+s) / 2}}
$$

in the space of $C^{\infty}$-functions on the circle. The functions $e^{i n \phi}$ are pairwise orthogonal with respect to this scalar product and

$$
<e^{i n \phi}, e^{i n \phi}>=\frac{\Gamma(n+(1+s) / 2)}{\Gamma(n+(1-s) / 2)}
$$

If $s>0$, then the integral (11) diverges. Nevertheless, the formula (2) makes sense and we can consider (2) as the definition of the divergent integral (11). Denote by $\mathcal{H}_{s}$ the completion of the space $C^{\infty}\left(\mathbb{T}^{1}\right)$ with respect to the scalar product (1). We have

$$
f(\phi)=\sum c_{n} e^{i n \phi} \in \mathcal{H}_{s} \Leftrightarrow \sum n^{s}\left|c_{n}\right|^{2}<\infty
$$

i.e. $\mathcal{H}_{s}$ is a Sobolev space on the circle $\mathbb{T}^{1}$.

We realize the group $S L(2, \mathbb{R})$ as the group of matrices $g=\left(\begin{array}{cc}a & b \\ \bar{b} & \bar{a}\end{array}\right)$ satisfying the condition $|a|^{2}-|b|^{2}=1$. Define a representation of the group 
$S L(2, \mathbb{R})$ in the space $\mathcal{H}_{s}$ by the formula

$$
T_{s}\left(\begin{array}{cc}
a & b \\
\bar{b} & \bar{a}
\end{array}\right) f(z)=f\left(\frac{a z+b}{\bar{b} z+\bar{a}}\right)|\bar{b} z+\bar{a}|^{s-1} .
$$

The representations $T_{s}$ are unitary in the Hilbert space $\mathcal{H}_{s}$ (this fact is simple but it is not trivial). They are called the complementary series representations of $S L(2, \mathbb{R})$. Note that the representations $T_{s}$ and $T_{-s}$ are equivalent.

Consider the tensor product $T_{s_{1}} \otimes T_{s_{2}}$ of two complementary series representations. The space $\mathcal{H}_{s_{1}} \otimes \mathcal{H}_{s_{2}}$ is a space of functions on the two-dimensional torus $\mathbb{T}^{2}: z_{1}=e^{i \phi_{1}}, z_{2}=e^{i \phi_{2}}$ equipped with the scalar product

$$
\begin{aligned}
& <f_{1}, f_{2}> \\
= & \int_{0}^{2 \pi} \int_{0}^{2 \pi} \int_{0}^{2 \pi} \int_{0}^{2 \pi} \frac{f_{1}\left(\phi_{1}, \phi_{2}\right) \overline{f_{2}\left(\psi_{1}, \psi_{2}\right)}}{\left|\sin \left(\phi_{1}-\psi_{1}\right) / 2\right|^{\left(1+s_{1}\right) / 2}\left|\sin \left(\phi_{2}-\phi_{2}\right) / 2\right|^{\left(1+s_{2}\right) / 2}}
\end{aligned}
$$

The group $S L_{2}(\mathbb{R})$ acts in this space via the formula

$$
\left(T_{s_{1}} \otimes T_{s_{2}}\right)\left(\begin{array}{cc}
a & b \\
\bar{b} & \bar{a}
\end{array}\right) f\left(z_{1}, z_{2}\right)=f\left(\frac{a z_{1}+b}{\bar{b} z_{1}+\bar{a}}, \frac{a z_{2}+b}{\bar{b} z_{2}+\bar{a}}\right)\left|\bar{b} z_{1}+\bar{a}\right|^{s_{1}-1}\left|\bar{b} z_{2}+\bar{a}\right|^{s_{2}-1}
$$

\subsection{Restriction to the diagonal.}

Denote by $\Delta$ the diagonal $\phi_{1}=\phi_{2}$ of the torus $\mathbb{T}^{2}$.

Theorem 1.1. Let $s_{1}+s_{2}>1$. Then the restriction operator restricting a function $f \in C^{\infty}\left(\mathbb{T}^{2}\right)$ to the diagonal $\Delta$ extends to a bounded operator $R$ from the space $\mathcal{H}_{s_{1}} \otimes \mathcal{H}_{s_{2}}$ to the space $L^{2}(\Delta)$.

We emphasize that the functions $f \in \mathcal{H}_{s_{1}} \otimes \mathcal{H}_{s_{2}}$ may be discontinuous and hence a function $f \in \mathcal{H}_{s}$ has no value in a individual point of the torus. Nevertheless, the restriction operator $R$ is well defined.

Proof. The space $\mathcal{H}_{s_{1}} \otimes \mathcal{H}_{s_{2}}$ consists of functions

$$
f\left(\phi_{1}, \phi_{2}\right)=\sum c_{n, m} e^{i n \phi_{1}} e^{i m \phi_{2}}
$$

satisfying the condition

$$
\sum(1+|n|)^{s_{1}}(1+|m|)^{s_{2}}\left|c_{n, m}\right|^{2}<\infty
$$

The restriction of $f$ to the diagonal $\Delta$ has the form

$$
\sum_{k}\left(\sum_{m+n=k} c_{m, n}\right) e^{i k \phi}
$$

and the convergence of the series $\sum c_{m, n}$ easily can be deduced from the CauchyBunyakovsky inequality.

The restriction operator is a bounded $S L(2, \mathbb{R})$-interwining operator from $T_{s_{1}} \otimes T_{s_{2}}$ to $T_{s_{1}+s_{2}-1}$. The representation $T_{s_{1}} \otimes T_{s_{2}}$ is unitary and hence $T_{s_{1}+s_{2}-1}$ is a subrepresentation of $T_{s_{1}} \otimes T_{s_{2}}$. 
1.3. Dual picture: distributions supported on the diagonal. Now let $s_{1}+s_{2}<-1$ (recall that $T_{-s} \simeq T_{s}$ ). It turns out that in this case the Hilbert space $\mathcal{H}_{s_{1}} \otimes \mathcal{H}_{s_{2}}$ contains distributions supported on the diagonal $\Delta$. Obviously the space of distributions supported on the diagonal is an invariant subspace. It is easy to show that the representation of $S L(2, \mathbb{R})$ in this subspace is equivalent to $T_{s_{1}+s_{2}+1}$ and we again obtain an embedding of a complementary series repesentation into the tensor product.

1.4. Separation of a discrete increment. Let $s_{1}=s_{2}=s<-1 / 2$. Consider the operator

$$
J f\left(\phi_{1}, \phi_{2}\right)=\left|\sin \left(\frac{\phi_{1}-\phi_{2}}{2}\right)\right|^{s} f\left(\phi_{1}, \phi_{2}\right)
$$

Then the operator $J$ interwines the representation $T_{s} \otimes T_{s}$ and the representation

$$
\left(T_{0} \otimes T_{0}\right)\left(\begin{array}{cc}
a & b \\
\bar{b} & \bar{a}
\end{array}\right) f\left(z_{1}, z_{2}\right)=f\left(\frac{a z_{1}+b}{\bar{b} z_{1}+\bar{a}}, \frac{a z_{2}+b}{\bar{b} z_{2}+\bar{a}}\right)\left|\bar{b} z_{1}+\bar{a}\right|^{-1}\left|\bar{b} z_{2}+\bar{a}\right|^{-1} .
$$

The last representation is the standard representation of $S L(2, \mathbb{R})$ in $L^{2}\left(\mathbb{T}^{2}\right)$. The kernel of $J$ consists of distributions supported on the diagonal and the image is dense in $L^{2}$. Hence the representation $T_{s} \otimes T_{s}$ is equivalent to the direct sum of the representation $T_{1+2 s}$ and the representation of $S L(2, \mathbb{R})$ in $L^{2}$ on the torus.

Remark. The operator $J$ is a operator of multiplication on nonsmooth function in space of distributions. This operator is unbounded and hence it is nessesary to be careful, see [NO], $\S 7$ for details and for the case $s_{1} \neq s_{2}$.

Remark. The existence of the embedding of $T_{s_{1}+s_{2}-1}$ into $T_{s_{1}} \otimes T_{s_{2}}$ was obtained by Pukanzky [Puk] in 1961, and an analogous result for $S L(2, \mathbb{C})$ was obtained by Naimark [Nai] (1961). The explanation of this phenomenon using the restriction operator wasn't observed before [Ner1] (1986).

\section{Hilbert spaces defined by positive defined ker- nels.}

2.1. Positive definite kernels. Let $\Xi$ be a set. A function $K(x, y)$ on $\Xi \times \Xi$ is called a positive definite kernel if for each finite collection of points $x_{1}, \ldots, x_{n} \in \Xi$ we have

$$
\operatorname{det}\left(\begin{array}{cccc}
K\left(x_{1}, x_{1}\right) & K\left(x_{1}, x_{2}\right) & \ldots & K\left(x_{1}, x_{n}\right) \\
K\left(x_{2}, x_{1}\right) & K\left(x_{2}, x_{2}\right) & \ldots & K\left(x_{2}, x_{n}\right) \\
\vdots & \vdots & \ddots & \vdots \\
K\left(x_{n}, x_{1}\right) & K\left(x_{n}, x_{2}\right) & \ldots & K\left(x_{n}, x_{n}\right)
\end{array}\right) \geq 0
$$

To each positive definite kernel $K(x, y)$ we associate a Hilbert space $H$ and fixed system of vectors $\Psi_{x} \in H$ enumerated by $x \in \Xi$ such that 
1. The linear span of the system $\Psi_{x}$ is dense in $H$

2. For all $x, y \in \Xi$ we have

$$
<\Psi_{y}, \Psi_{x}>_{H}=K(x, y)
$$

The system $\Psi_{x}$ is called a supercomplete basis or an overfull system.

Let $h \in H$. We consider the function $f_{h}(x)=J h(x)$ on $\Xi$ given by formula

$$
J F(x)=f_{h}(x)=<h, \Psi_{x}>
$$

where $x \in \Xi$.

We will identify the space $H$ and the image of the operator $J$. In other words we consider the Hilbert space $H$ as a space of functions on $\Xi$.

2.2. Hilbert spaces of holomorphic functions. Let $\Omega$ be an open domain in $\mathbb{C}^{n}$. A function $K(z, u)$ on $\Omega \times \Omega$ is called a reproducing kernel on $\Omega$ if

1. $K(z, u)$ is a positive definite kernel

2. The function $K(z, u)$ is holomorphic with respect to the variable $z$ and antiholomorphic with respect to the variable $u$.

Consider the associated Hilbert space $H$ and let us realize it as a space of functions on $\Omega$ by the formula (何. It is easy to see that the functions $f_{h}$ are holomorphic, i.e. the Hilbert space $H$ can be considered as a Hilbert space of holomorphic functions on $\Omega$.

2.3. Example: Berezin spaces on Cartan domains. Let $p \leq q$. Denote by $B_{p, q}$ the space of all $p \times q$-matrices $z$ satisfying the condition $\|z\|<1$, i.e $B_{p, q}$ is a Cartan domain of the first type.

The pseudounitary group $U(p, q)$ consists of $(p+q) \times(p+q)$-matrices $g=$ $\left(\begin{array}{ll}a & b \\ c & d\end{array}\right)$ satisfying the condition

$$
g \cdot\left(\begin{array}{cc}
1 & 0 \\
0 & -1
\end{array}\right) \cdot g^{*}=\left(\begin{array}{cc}
1 & 0 \\
0 & -1
\end{array}\right)
$$

The group $U(p, q)$ acts on $B_{p, q}$ by the fractional-linear transformations

$$
z \mapsto z^{[g]}:=(a+z c)^{-1}(b+z d)
$$

The stabilizer of the point $z=0$ consists of matrices having the form $\left(\begin{array}{ll}a & 0 \\ 0 & d\end{array}\right)$, where $a \in U(p), d \in U(q)$. Hence $B_{p, q}$ is the Riemannian symmetric space

$$
U(p, q) /(U(p) \times U(q))
$$

Theorem 2.1. (see [Ber1], see also [Wal]) Let $s=0,1,2, \ldots, p-1$ or $s>p-1$. Then the kernel

$$
K_{s}(z, u)=\operatorname{det}^{-s}\left(1-z u^{*}\right)
$$


is positive definite on $B_{p, q}$.

Denote by $V_{s}$ the Hilbert space of holomorphic functions on $B_{p, q}$ defined by the kernel $K_{s}(z, u)$ (for a discussion of this space see for instance [Ber1], [NO]). The group $U(p, q)$ acts on $V_{s}$ by the unitary operators

$$
T_{s}\left(\begin{array}{ll}
a & b \\
c & d
\end{array}\right) f(z)=f\left(z^{[g]}\right) \operatorname{det}^{-s}(a+z c),
$$

where $z^{[g]}$ is given by the formula (6).

The representations $T_{s}$ are called spherical highest weight representations of $U(p, q)$.

Remark. If $s$ is an integer, then $T_{s}$ is a representation of the group $U(p, q)$ itself. If $s$ is not an integer, then the function $\operatorname{det}^{-s}(\cdot)$ is multivalued and hence $T_{s}$ is a representation of the universal covering group of $U(p, q)$ (in other words, the representation $T_{s}$ is a projective representation of the group $\left.U(p, q)\right)$.

Remark. If $s>p-1$, then the space $V_{s}$ contains all polynomial functions on the Cartan domain $B_{p, q}$. If $s=0,1, \ldots, p-1$, then all functions $f \in V_{s}$ satisfy the following system of partial differential equations:

$$
\left[\operatorname{det}\left(\begin{array}{ccc}
\frac{\partial}{\partial z_{i_{1} j_{1}}} & \cdots & \frac{\partial}{\partial z_{i_{\alpha} j_{1}}} \\
\vdots & \ddots & \vdots \\
\frac{\partial}{\partial z_{i_{1} j_{\alpha}}} & \cdots & \frac{\partial}{\partial z_{i_{\alpha} j_{\alpha}}}
\end{array}\right)\right] f(z)=0
$$

for all $\alpha=2,3, \ldots, s+1$ and all $i_{\mu}, j_{\nu}$ satisfying the inequalities $0 \leq i_{\mu} \leq q$, $0 \leq j_{\nu} \leq p$.

2.4. Cartan domains of second and third types. The Cartan domain $C_{n}$ of third type is the set of all $n \times n$ symmetric matrices $z$ with complex coefficients satisfying the condition $\|z\|<1$. The group $G$ of biholomorphic transformations (automorphisms) of the domain $C_{n}$ consists of transformations having the form (6), where a matrix $g=\left(\begin{array}{ll}a & b \\ c & d\end{array}\right)$ satisfies the condition

$$
\left(\begin{array}{ll}
a & b \\
c & d
\end{array}\right)\left(\begin{array}{cc}
1 & 0 \\
0 & -1
\end{array}\right)\left(\begin{array}{cc}
a & b \\
c & d
\end{array}\right)^{t}=\left(\begin{array}{cc}
1 & 0 \\
0 & -1
\end{array}\right) ; \quad d=\bar{a}, \quad c=\bar{b} .
$$

It is easy to see that the group $G$ is isomorphic to the real symplectic group $S p(2 n, \mathbb{R})$ and the domain $C_{n}$ is the Riemannian symmetric space

$$
C_{n}=S p(2 n, \mathbb{R}) / U(n) .
$$

The kernel

$$
K_{s}(z, u):=\operatorname{det}^{-s}(1-z \bar{u})
$$

is positive definite if and only if

$$
\{s=0,1 / 2, \ldots,(n-1) / 2\} \text { or }\{s>(n-1) / 2\} .
$$


The Cartan domain $D_{n}$ of the second type is the set of all $n \times n$ skewsymmetric matrices $z$ satisfying the condition $\|z\|<1$. The group $G$ of automorphisms of the domain $D_{n}$ is the group of transformations (6), where the matrix $g=\left(\begin{array}{ll}a & b \\ c & d\end{array}\right)$ satisfies the condition

$$
\left(\begin{array}{ll}
a & b \\
c & d
\end{array}\right)\left(\begin{array}{cc}
0 & 1 \\
1 & 0
\end{array}\right)\left(\begin{array}{cc}
a & b \\
c & d
\end{array}\right)^{t}=\left(\begin{array}{cc}
0 & 1 \\
1 & 0
\end{array}\right) ; \quad d=\bar{a}, \quad c=\bar{b} .
$$

Remark. Condition (9) implies (5).

The group $G$ is isomorphic to the real classical group $S O^{*}(2 n)$ and the domain $D_{n}$ is the symmetric space

$$
D_{n}=S O^{*}(2 n) / U(n) .
$$

The kernel $K(z, u)=\operatorname{det}^{-s}\left(1-z u^{*}\right)$ on $D_{n}$ is positive definite if $s=0,1,2, \ldots$, $n-1$ or $s>n-1$.

Spherical highest weight representations of $S p(2 n, \mathbb{R})$ and $S O^{*}(2 n)$ act in Hilbert spaces associated to the kernels $K_{s}$ by the formula (7).

2.5. Matrix-valued positive definite kernels. Consider a set $\Xi$ and a finite dimensional complex euclidian space $\mathcal{Y}$. Denote by $G L(\mathcal{Y})$ the group of invertible linear operators in $\mathcal{Y}$. We say that a function

$$
L: \Xi \times \Xi \rightarrow G L(\mathbb{C}, \mathcal{Y})
$$

is a matrix-valued positive definite kernel (see for instance [HN]) if the function

$$
\widetilde{L}((z, \xi) ;(u, \eta)):=<L(z, u) \xi, \eta>; \quad(z, \xi),(u, \eta) \in \Xi \times \mathcal{Y}
$$

is a positive definite kernel on $\Xi \times \mathcal{Y}$.

Suppose we have a matrix-valued positive defined kernel on $\Xi$. Then there exists a unique Hilbert space $H$ and a map $\Psi: \Xi \times \mathcal{Y} \rightarrow H$ such that

a) The map $\Psi$ is linear on each fiber $z \times \mathcal{Y} \subset \Xi \times \mathcal{Y}$.

b) $<\Psi(z, \xi), \Psi(u, \eta)>_{H}=<L(z, u) \xi, \eta>_{\mathcal{Y}}$

c) The image of the map $\Psi$ is total in $H$.

Again there exists a canonical map $J$ from the Hilbert space $H$ to the space of $\mathcal{Y}$-valued functions on $\Xi$. Let $h \in H$. Then the function $f_{h}=J f$ is defined via the equation

$$
<f_{h}(z), \xi>\mathcal{Y}=<h, \Psi(z, \xi)>_{H} \text {. }
$$

2.6. Highest weight representations of the groups $G=U(p, q)$, $S p(2 n, \mathbb{R}), S O^{*}(2 n)$.

Consider the case $G=U(p, q)$. Let $\rho=\rho_{1} \otimes \rho_{2}$ be an irreducible finitedimensional holomorphic representation of the group $G L(p, \mathbb{C}) \times G L(q, \mathbb{C})$ or of 
its universal covering. Further let $W=W_{1} \otimes W_{2}$ be the space of the representation $\rho$. Assume that the function

$$
K_{\rho}(z, u):=\rho_{1}\left(1-z u^{*}\right) \otimes \rho_{2}\left(1-u^{*} z\right)
$$

is a matrix-valued positive-definite kernel (see [Ols2] for conditions of positive definiteness, see also [NO]). Consider the associated Hilbert space $H_{\rho}$ of $W_{1} \otimes$ $W_{2}$-valued holomorphic functions on $B_{p, q}$. Then the unitary highest weight representation $T_{\rho}$ of the group $U(p, q)$ (or of its universal covering) acts on the space $H_{\rho}$ by the formula

$$
T_{\rho}\left(\begin{array}{ll}
a & b \\
c & d
\end{array}\right) F(z)=\left(\rho_{1}(a+z c) \otimes \rho_{2}\left(b-d z^{[g]}\right)\right) F\left(z^{[g]}\right)
$$

Let $G=S p(2 n, \mathbb{R})$ or $S O^{*}(2 n)$. Consider a finite dimensional holomorphic representation $\rho$ of the group $G L(n, \mathbb{C})$ or of its universal covering group. Assume the matrix-valued kernel $K_{\rho}(z, u)=\operatorname{det}^{-s}\left(1-z u^{*}\right)$ to be positive-definite on the Cartan domain $C_{n}$ or $D_{n}$, respectively. Denote by $H_{\rho}$ the associated Hilbert space of holomorphic vector-valued functions. Then the unitary highest weight representation $T_{\rho}$ of the group $G$ is given by the formula

$$
T_{\rho}\left(\begin{array}{ll}
a & b \\
c & d
\end{array}\right) f(z)=\rho(a+z c) F\left(z^{[g]}\right) .
$$

\subsection{Bibliographical comments.}

a) There are also Cartan domains of 4 -th type $S O(n, 2) /(S O(n) \times O(2))$ and two exceptional Cartan domains. There exist associated highest weight representations. The Cartan domains $S O(n, 2) /(O(n) \times O(2))$ are a popular object of complex analysis ( tube of future), see [VS]. These domains often are called Lie spheres or Lie balls, but this term is a result of a philological mistake.(Lie sphere is a oriented sphere, oriented plane or point in $\mathbb{R}^{n}$. The space of all Lie spheres is a homogeneous space $O(n+2) /(O(n) \times O(2))$ (see for instance $[\mathrm{Cec}])$. The term 'Lie sphere' for a homogeneus space $O(n, 2) /(S O(n) \times O(2))$ appeared in russian translation of famous chinese book [Hua]. It is quite strange to name this space by 'sphere' and it was transformed to 'ball'.)

b) Conditions for the positive-definiteness of the standard scalar-valued kernels on Cartan domains of types one through four were discovered by Berezin [Ber] (with some additions given by Gindikin [Gin]); Berezin and Gindikin didn't consider exceptional domains; later conditions for the positive-definiteness in the general case (including exceptional) were obtained by Wallach [Wal], Rossi - Vergne [RV] and Jackobsen [Jac], proofs of these authors are essentially different).

c) Conditions for the positive-definiteness of the standard kernels for $B_{p, q}$ were obtained in [Ols2]. For other domains this problem was discussed by many authors, the final result was obtained in [EHW]. 


\section{Boundary values of holomorphic functions.}

\section{1. $L^{1}$-limits.}

Let $\Omega \subset \mathbb{C}^{n}$ be an open domain, $\partial \Omega$ its boundary, and $\bar{\Omega}$ be the closure of $\Omega$. We say that $\Omega$ is a regular circle domain if

a) for all $z \in \Omega$ and $\lambda \in \mathbb{C}$ such that $|\lambda| \leq 1$ we have $\lambda z \in \Omega$

b) for all $z \in \partial \Omega$ and $\lambda \in \mathbb{C}$ such that $|\lambda|<1$ we have $\lambda z \in \Omega$.

Let $K(z, u)$ be a reproducing kernel in $\Omega$ satisfying the condition

$$
K\left(e^{i \phi} z, e^{i \phi} u\right)=K(z, u)
$$

and $H$ be the Hilbert space of holomorphic functions associated to this kernel.

Remark. Cartan domains are regular circle domains and the Berezin kernels satisfy the condition (11).

Theorem 3.1. (see [NO]) Let $M \subset \partial \Omega$ be a compact subset. Let $\mu$ be a measure supported on $M$. We make the following assumptions

a) $K^{*}(z, u):=\lim _{c \rightarrow 1-0} K(c z, c u)$ exists almost everywhere on $M \times M$ with respect to the measure $\mu \times \mu$.

b) $K^{*} \in L^{1}(M \times M, \mu \times \mu)$ and $\lim _{c \rightarrow 1-0} K(c z, c u)$ is dominated, i.e. there exists a function $S(z, u) \in L^{1}(M \times M, \mu \times \mu)$ such that $|K(c z, c u)|<S(z, u)$ almost everywhere on $M \times M$.

Then the restriction operator restricting a function $f \in H$ to the set $M$ is a well-defined operator

$$
H \rightarrow L^{1}(M, \mu) .
$$

This theorem doesn't cover all cases in which the restriction operator exists (see Subsections 3.2-3.3 below). Hence the following natural problem arises.

Problem 1. Let $\Omega \subset \mathbb{C}^{n}$ be an open domain. Let $K(z, u)$ be a reproducing kernel and let $H$ be the associated Hilbert space. Further let $M$ be a submanifold of the Shilov boundary of $\Omega$. Find conditions for the existence of a restriction operator from $H$ to some Hilbert space of functions on M.

Remark. The case which is interesting for harmonic analysis is the following. Let the domain $\Omega$ be a Cartan domain $G / K$ and $H=V_{s}$ be a Berezin space. Consider a subgroup $Q \subset G$ and some $Q$-orbit $M$ in the Shilov boundary of the domain $\Omega$. Suppose the restriction operator restricting functions $f \in H_{s}$ to the manifold $M$ exists. Then we obtain an interwining operator from the Berezin space $H_{s}$ to some Hilbert space $H(M)$ of functions on $M$. The representation of $Q$ in $H(M)$ is equivalent to a subrepresentation of the representation of $Q$ in $H$. If the group $Q$ is semisimple and the orbit $M$ is compact, then the representation of $Q$ in $H(M)$ has purely discrete spectrum.

3.2. Restrictions of functions of polynomial growth on a ball. Denote by $B_{q}$ the unit ball

$$
\left|z_{1}\right|^{2}+\ldots+\left|z_{q}\right|^{2}<1
$$


in $\mathbb{C}^{q}$ and let $H^{\infty}\left(B_{q}\right)$ be the Hardy space of bounded holomorphic functions on $B_{q}$. Denote by $\langle\cdot, \cdot\rangle$ the standard scalar product in $\mathbb{C}^{n}$.

Consider a $C^{1}$-curve $\gamma(t)$ in the sphere $\partial B_{q}=S^{2 q-1}$.

Theorem 3.2 (see $[\mathrm{NR}],[\mathrm{Rud}])$ Let $\gamma(t)$ satisfy the condition

$$
\forall t: \operatorname{Im}\left\langle\gamma(t), \gamma^{\prime}(t)\right\rangle \neq 0
$$

Then for each $f \in H^{\infty}\left(B^{q}\right)$ the nontangential limit $f(z)$ as $z \rightarrow \gamma(t)$ exists almost everywhere on the curve $\gamma(t)$.

Remark. Obviously the vector $\gamma^{\prime}(t)$ is tangent to the sphere $S^{2 q-1}$. Hence we have $\operatorname{Re}\left\langle\gamma(t), \gamma^{\prime}(t)\right\rangle=0$ for all $t$. Fix $z \in S^{2 q-1}$ and let $v \in \mathbb{C}^{q}$ be a vector. The equation $\langle z, v\rangle=0$ defines a subspace of codimension 1 in the tangent space to the sphere $S^{2 q-1}$ in the point $z \in S^{2 q-1} \subset \mathbb{C}^{n}$. We obtain a contact distribution on the sphere (see [Rud], 5.4, 10.5). The condition (12) means that $\gamma(t)$ is transversal to this distribution.

Denote by $\mathcal{D}^{\prime}$ the space of all holomorphic functions of polynomial grouth in $B^{q}$ :

$$
f \in \mathcal{D}^{\prime} \Leftrightarrow \exists N: \sup |f(z)|\left(1-|z|^{2}\right)^{N}<\infty
$$

Remark. The space $\mathcal{D}^{\prime}$ is a union $\mathcal{D}^{\prime}=\cup_{s>0} V_{s}\left(B_{q}\right)$ of Berezin spaces on the ball, see Subsection 2.3. The space $V_{q}\left(B_{q}\right)$ coincides with the Hardy space $H^{2}\left(B_{q}\right)$. The space $V_{q+1}\left(B_{q}\right)$ is the Bergman space (i.e the intersection of $L^{2}\left(B_{q}\right)$ with the space of holomorphic functions).

Remark. It is well known (and more or less obvious) that each function $f \in \mathcal{D}^{\prime}$ has a limit on the boundary in the sense of distributions (see [RS],IX.3 for a discussion of theorems of this type and further references).

Denote by $R$ the restriction operator resctricting a holomorphic function $f$ on the ball $B_{q}$ to the curve $\gamma(t)$. Obviously the function $R f$ is well-defined if the function $f$ is continuous up to the boundary.

Theorem 3.3. (see [Ner2]) Let $\gamma(t)$ be a $C^{\infty}$-smooth curve in $\partial B^{q}$ satisfying the condition (17). Then the restriction operator $R$ restricting a holomorphic function to $\gamma(t)$ extends to a bounded operator from $\mathcal{D}^{\prime}$ to the space of distributions on $\gamma(t)$.

3.3. Restriction of functions of polynomial growth on a polydisc.

Denote by $P^{n}$ the polydisc $\left|z_{1}\right|<1, \ldots,\left|z_{n}\right|<1$. Let $\mathbb{T}^{n}$ be the torus $z_{1}=e^{i \phi_{1}}, \ldots, z_{n}=e^{i \phi_{n}}$. Let $\gamma(t)=\left(\phi_{1}(t), \ldots, \phi_{n}(t)\right)$ be a time-like $C^{\infty}$-curve in $\mathbb{T}^{n}$ i.e.

$$
\forall t: \phi_{1}^{\prime}(t)>0, \ldots, \phi_{n}^{\prime}(t)>0
$$

Denote by $\mathcal{D}^{\prime}$ the space of holomorphic functions of polynomial growth in $P^{n}$, i.e

$$
f \in \mathcal{D}^{\prime} \Leftrightarrow \exists N: \sup f(z) \prod_{k=1}^{n}\left(1-\left|z_{k}\right|\right)^{N}<\infty
$$


This condition is equivalent to the existence of an $L>0$ with

$$
\begin{gathered}
f\left(z_{1}, \ldots, z_{n}\right)=\sum c_{k_{1}, \ldots, k_{n}} z_{1}^{k_{1}} \ldots z_{n}^{k_{n}} \in \mathcal{D}^{\prime} \quad \text { and } \\
\sum \frac{\left|c_{k_{1}, \ldots, k_{n}}\right|}{\left[\left(k_{1}+1\right) \ldots\left(k_{n}+1\right)\right]^{L}}<\infty
\end{gathered}
$$

Theorem 3.4. (see [Ner2]) The restriction operator $R$ restricting a holomorphic function $f$ on $P^{n}$ to the curve $\gamma(t)$ extends to a bounded operator from the space $\mathcal{D}^{\prime}$ to the space of distributions on the curve $\gamma(t)$.

Example. Consider a bidisc $P^{2}$ and the Hilbert space $H_{s_{1}, s_{2}}$ associated to the reproducing kernel

$$
\left(1-z_{1} \bar{u}_{1}\right)^{-s_{1}}\left(1-z_{2} \bar{u}_{2}\right)^{-s_{2}},
$$

where $s_{1}, s_{2}>0$. It is easy to show that $\mathcal{D}^{\prime}=\cup_{s_{1}, s_{2}>0} H_{s_{1}, s_{2}}$. If $\gamma(t)$ has the form $\left(\gamma_{1}(t), \gamma_{2}(t)\right)=\left(e^{i t}, e^{i \sigma}\right)$ where $\sigma=$ const, then a restriction operator to $\gamma(t)$ doesn't exist for all $s_{1}, s_{2}$. If $\gamma(t)$ satisfies the condition $\gamma_{1}^{\prime}(t)>0, \gamma_{2}^{\prime}(t)<0$ then the restriction operator exists only in the case $s_{1}+s_{2}<1$.

3.4. A conjecture. It seems to me that Theorems 3.3-3.4 are partial cases of some general fact. Let $\Omega \subset \mathbb{C}^{N}$ be an open domain. Let $M$ be a submanifold in the Shilov boundary of $\Omega$. Denote by $T_{m}$ the tangent space to $M$ in the point $m \in M$. We identify $T_{m}$ with a linear submanifold in $\mathbb{C}^{n}$. Denote by $S_{m}=i \cdot T_{m}$ the linear submanifold which consists of the vectors

$$
i \cdot(v-m)+m,
$$

where $v \in T_{m}$ (and $i^{2}=-1$ ). We say the submanifold $M$ is time-like if for each point $m \in M$ there exists an open cone $C_{m} \subset S_{m}$ with the vertex $m$ and an $\epsilon$-neighborhood $\mathcal{U}_{\epsilon}(m)$ of $m$ such that

$$
C_{m} \cap \Omega \supset \mathcal{U}_{\epsilon}(m) \cap C_{m} .
$$

Conjecture. Each holomorphic function of polynomial growth in $\Omega$ has a restriction to a time-like manifold $M$ in the Shilov boundary in the sense of distributions.

Theorems 3.3-3.4 are partial cases of this conjecture. It is also similiar to the standard facts on limits of functions of polynomial growth on the whole Shilov boundary mentioned above (see [RS], IX.3, [Vla], [VS]). Neverless I couldn't find this fact in the literature.

\section{Positive definite kernels on Riemannian non- compact symmetric spaces}

4.1. Spherical kernel-representations of the groups $U(p, q)$. Consider the matrix ball $B_{p, q}$, see Subsection 2.3. Let $s=0,1,2, \ldots, p-1$ or $s>p-1$. 
Consider the function

$$
L_{s}(z, u)=\left|\operatorname{det}\left(1-z u^{*}\right)\right|^{-2 s},
$$

where $z, u \in B_{p, q}$. With the notations of Subsection 2.3 we have

$$
L_{s}(z, u)=K_{s}(z, u) \overline{K_{s}(z, u)}
$$

and hence $L_{s}(z, u)$ is a positive definite kernel on $B_{p, q}$. Denote by $H_{s}$ the Hilbert space associated with the kernel $L_{s}(z, u)$. Using the formula (伍) we identify this space with some space of real analytic functions on $B_{p, q}$.

The group $U(p, q)$ acts on $H_{s}$ by the unitary operators

$$
A_{s}(g) f(z)=f\left(z^{[g]}\right)|\operatorname{det}(a+z c)|^{-2 s},
$$

where $z^{[g]}$ is defined by the formula (6).

Problem 2. Decompose the representation $A_{s}$

We will call the representations $T_{s}$ spherical kernel-representations of the group $U(p, q)$.

Some partial cases of this question were discussed in the end of the 70-ies, see [Ber2],[Rep],[Gut]. In fact, only the case where $s$ is large has been discussed there. In this case the most interesting phenomena don't appear. For a while such problems were more or less forgotten. During the last years some of these problems attracted interest again (see [NO], [OØ], [ØZ],[Dij]).

I would like to try to explain why this problem is interesting and also to discuss some approaches to this problem.

4.2. Limit as $s \rightarrow \infty$. We want to show that the limit of the kernelrepresentations as $s \rightarrow+\infty$ is the canonical representation of $U(p, q)$ in the space $L^{2}$ on Riemannian symmetric space $U(p, q) /(U(p) \times U(q))$.

Consider the system of vectors $\Psi_{z} \in H_{s}$ (see Subsection 2.2). Let $\chi$ be a distribution in $B_{p, q}$ with compact support. Consider the vector $\Theta(\chi) \in H_{s}$ defined by the equality

$$
\Theta(\chi)=\int_{B_{p, q}}\left|\operatorname{det}^{s}\left(1-z z^{*}\right)\right| \chi(z) \Psi_{z} d z d \bar{z}
$$

and the scalar product $\{\cdot, \cdot\}_{s}$ in the space of distributions on $B_{p, q}$ with compact support given by the formula

$$
\begin{aligned}
& \left\{\chi_{1}, \chi_{2}\right\}_{s}:=\left\langle\Theta\left(\chi_{1}\right), \Theta\left(\chi_{2}\right)\right\rangle \\
= & \int_{B_{p, q}} \int_{B_{p, q}}\left|\frac{\operatorname{det}\left(1-z z^{*}\right) \operatorname{det}\left(1-u u^{*}\right)}{\operatorname{det}^{2}\left(1-z u^{*}\right)}\right|^{s} \chi_{1}(z) \chi_{2}(u) d z d \bar{z} d u d \bar{u} .
\end{aligned}
$$

We can identify the space $H_{s}$ with the completion of the space of distributions with respect to the scalar product $\{\cdot, \cdot\}_{s}$. The group $U(p, q)$ acts on this space of distributions by the formula

$$
B_{s}(g) f(z)=f\left(z^{[g]}\right)
$$


(the formula doesn't depend on $s$, nevertheless, the scalar product and the spectrum of the representation do depend on $s$ in an essential way).

It appeared that for some normalizing multiplier $\omega(s)$ for all continuous functions $\phi_{1}(z), \phi_{2}(z)$ on $B_{p, q}$ with compact support we have

$$
\lim _{s \rightarrow+\infty} \omega(s)\left\{\phi_{1}, \phi_{2}\right\}_{s}=\int_{B_{p, q}} \phi_{1}(z) \phi_{2}(z) d z d \bar{z}
$$

4.3. Another formulation of the problem. Consider a spherical highest weight representation $T_{s}$ of the group $U(p, q)$ (see Subsection 2.3). Denote by $V_{s}$ the space of the representation $T_{s}$. Denote by $T_{s}^{*}$ the contragradient representation of $T_{s}$. Consider the tensor product $T_{s} \otimes T_{s}^{*}$. This representation acts on the space $V_{s} \otimes V_{s}$ of holomorphic functions on $B_{p, q} \times B_{p, q}$ via the operators

$$
\left(T_{s} \otimes T_{s}^{*}\right)(g)=f\left(z_{1}, z_{2}\right)=f\left(z_{1}^{[g]}, z_{2}^{[\bar{g}]}\right)\left(a+z_{1} c\right)^{-s}\left(\bar{a}+z_{2} \bar{c}\right)^{-s} .
$$

The $U(p, q)$-invariant scalar product in the space of holomorphic functions on $B_{p, q} \times B_{p, q}$ is defined by the reproducing kernel

$$
M\left(z_{1}, z_{2} ; u_{1}, u_{2}\right)=\operatorname{det}^{-s}\left(1-z_{1} u_{1}^{*}\right) \operatorname{det}^{-s}\left(1-z_{2} u_{2}^{*}\right)
$$

Consider the operator

$$
I: V_{s} \otimes V_{s} \rightarrow H_{s}
$$

defined by the formula

$$
\text { If }(z)=f(z, \bar{z})
$$

Obviously $I$ is a unitary operator interwining the representations

$$
T_{s} \otimes T_{s}^{*} \leftrightarrow A_{s}
$$

Hence we can formulate our problem in the form:

Problem 2'. Decompose the tensor product $T_{s} \otimes T_{s}^{*}$.

4.4. Orbits of the group $U(p, q)$ on the Shilov boundary.

Denote by $M_{p, q}$ the Shilov boundary of $B_{p, q}$. The elements of $M_{p, q}$ are matrices $z$ satisfying the condition

$$
z \cdot z^{*}=1
$$

In the other words, $z$ is the matrix of an isometric embedding $\mathbb{C}^{p} \rightarrow \mathbb{C}^{q}$. Hence $M_{p, q}$ is a complex Stiefel manifold.

The Shilov boundary of $B_{p, q} \times B_{p, q}$ is $M_{p, q} \times M_{p, q}$. The group $U(p, q)$ has $(p+1)$ orbits on $M_{p, q} \times M_{p, q}$. The only invariant of an orbit is the number

$$
\alpha=r k(z-\bar{u})
$$


i.e if $\left(z_{1}, u_{1}\right),\left(z_{2}, u_{2}\right) \in M_{p, q} \times M_{p, q}$ and $r k\left(z_{1}-\bar{u}_{1}\right)=r k\left(z_{2}-\bar{u}_{2}\right)$, then the points $\left(z_{1}, u_{1}\right),\left(z_{2}, u_{2}\right)$ belong to the same orbit.

We denote by $\Xi_{\alpha}$ the orbit corresponding to a given invariant $\alpha$.

Remark. The orbit $\Xi_{0}$ is compact, whereas the orbit $\Xi_{p}$ is open (in the Shilov boundary). For all $\alpha$ the closure of $\Xi_{\alpha}$ is $\cup_{\sigma \leq \alpha} \Xi_{\sigma}$.

4.5. Restriction of holomorphic functions to $U(p, q)$-orbits in the Shilov boundary. Fix an orbit $\Xi_{\alpha}$ of $U(p, q)$ in the Shilov boundary of $B_{p, q} \times$ $B_{p, q}$. It can happen (and it really does happen) that for small $s$ the function $f \in H_{s}=V_{s} \otimes V_{s}$ has a well defined restriction to the orbit $\Xi_{\alpha}$. In this case the restriction operator is an interwining operator from $V_{s} \times V_{s}$ to some Hilbert space of functions on $\Xi_{\alpha}$.

It can also happen (and it really does happen) that for small $s$ all first partial derivatives of the function $f \in H_{s}=V_{s} \otimes V_{s}$ have a well defined restriction to the orbit $\Xi_{\alpha}$ etc. (see discussion of this phenomenon in [NO], Section 7).

Fix $s$. For each $\alpha=0,1, \ldots, p-1$ consider the maximal number $\tau_{\alpha}$ such that all partial derivatives of order $\leq \tau_{\alpha}$ of functions $f \in V_{s} \otimes V_{s}$ have welldefined restrictions to $\Xi_{\alpha}$. (these numbers aren't known, but Theorem 3.1 gives a possibility to estimate them; I don't know whether these estimates are best possible or not). If restrictions of the functions $f \in H_{s}=V_{s} \otimes V_{s}$ to $\Xi_{\alpha}$ don't exist we suppose $\tau_{\alpha}=-1$.

Remark. For large $s$ the restriction operators don't exist, i.e. we have $\tau_{\alpha}=-1$ for all $\alpha<p-1$ (see below subsection 4.6).

Fix $\alpha=0,1, \ldots p-1$. Consider some $i_{\alpha}=1, \cdots, \tau_{\alpha}$. Denote by $Q\left[\alpha, i_{\alpha}\right]$ the space of functions $f \in V_{s} \otimes V_{s}$ such that all partial derivatives of $f$ of orders $\leq i$ equal zero on $\Xi_{\alpha}$. We obtain a filtration

$$
\begin{gathered}
0 \subset Q\left[p-1, \tau_{p-1}\right] \subset Q\left[p-1, \tau_{p-1}-1\right] \subset \ldots \subset Q[p-1,0] \subset \\
\subset Q\left[p-2, \tau_{p-2}\right] \subset \ldots \subset Q[p-2,1] \subset Q[p-2,0] \subset \ldots \\
\ldots \subset Q\left[0, \tau_{0}\right] \subset \ldots Q[0,1] \subset Q[0,0] \subset V_{s} \otimes V_{s}
\end{gathered}
$$

Remark. For large $s$ this filtration is trivial, but for small $s$ it is quite long.

Consider the representations of $U(p, q)$ in the subquotients of this filtration. Obviously $A_{s}=T_{s} \otimes T_{s}^{*}$ is equivalent to the direct sum of the subquotients of the filtration $(15)$.

Remark. The representations of $U(p, q)$ in the subquotients have simple interpretations. For instance $\left(V_{s} \otimes V_{s}\right) / Q[0,0]$ is a subspace in the space of functions on the orbit $\Xi_{0}$. The space $Q[0,1] / Q[0,0]$ is a subspace in the space of sections of the normal bundle of the orbit $\Xi_{0}$. The space $Q[0,1] / Q[0,2]$ is a subspace in the space of sections of the symmetric square of the normal bundle etc., see the discussion in $[\mathrm{NO}], \S 7$.

It is natural to hope that the spectrum in each subquotient is more or less 'uniform', i.e. that the orbit structure of the Shilov boundary gives a separation of the quite complicated spectrum of $A_{s}$ into different types (compare with the [GG]-project ). 
4.6. Large $s$. If $s$ is large enough, then the restriction operators don't exist. In this case the representation $A_{s}$ is equivalent to the standard representation of the group $U(p, q)$ in $L^{2}$ on the Riemannian symmetric space $U(p, q) /(U(p) \times$ $U(q))$, see [Ber2], [Rep], [Gut], [O $\varnothing]$. A sufficient (but not nessessary) condition for this is $s>p+q-1$ (i.e. $T_{s}$ belongs to the Harish-Chandra discrete series).

4.6. Restriction to the compact orbit. The part of the spectrum which corresponds to the compact orbit $\Xi_{0}$ is purely discrete and it consists of quite exotic representations of $U(p, q)$. This part of the spectrum is not empty for small $s$ (for $s<(q-2 p-1) / 4)$. In [NO] one can find a description of the analogous situation for the compact orbit of $O(p, q)$ in the Shilov boundary of $B_{p, q}$.

In particular this gives a relatively simple way to construct singular unitary representations of the groups $U(p, q)$, see [NO].

4.7. Spherical kernel-representations of other classical groups All classical Riemannian noncompact symmetric spaces $G / K$ (up to the center of $G)$ can be realized as (real, complex or quaternionic) matrix balls (see [Ner3]). Namely the space $G / K$ is the space of matrices $z$ over the field $\mathbb{K}=\mathbb{R}, \mathbb{C}, \mathbb{H}$ (see below) satisfying the additional condition (see below) such that $\|z\|<1$.

We list the symmetric spaces $G / K$, fields and additional conditions.

$1^{*}$. $U(p, q) /(U(p) \times U(q))$ is the space of $p \times q$-matrices over $\mathbb{C}$.

$2^{*}$. $S p(2 n, \mathbb{R}) / U(n)$ is the space of symmetric $n \times n$-matrices over $\mathbb{C}$.

$3^{*}$. $S O^{*}(2 n) / U(n)$ is the space of skew symmetric $n \times n$-matrices over $\mathbb{C}$.

$4^{*}$. $O(p, q) /(O(p) \times O(q))$ is the space of $p \times q$-matrices over $\mathbb{R}$.

$5^{*}$. $G L(n, \mathbb{R}) / O(n)$ is the space of symmetric $n \times n$-matrices over $\mathbb{R}$.

$6^{*} . O(n, \mathbb{C}) / O(n)$ is the space of skew-symmetric $n \times n$-matrices over $\mathbb{R}$.

$7^{*} . G L(n, \mathbb{C}) / U(n)$ is the space of hermitian $n \times n$-matrices over $\mathbb{C}$.

$8^{*} . S p(p, q) /(S p(p) \times S p(q))$ is the space of $p \times q$-matrices over $\mathbb{H}$.

$9^{*}$. $G L(n, \mathbb{H}) / S p(p, q)$ is the space of hermitian $n \times n$-matrices over $\mathbb{H}$.

$10^{*}$. $S p(2 n, \mathbb{C}) / S p(n)$ is the space of skew hermitian (i.e. $\left.z=-z^{*}\right) n \times n$ matrices over $\mathbb{H}$.

In all cases enumerated above the group $G$ acts on the matrix ball $G / K$ by fractional-linear transformations

$$
z \mapsto z^{[g]}:=(a+z c)^{-1}(b+z d)
$$

Now let us consider positive definite kernels on $G / K$ having the form

$$
L_{s}(z, u)=\left|\operatorname{det}\left(1-z u^{*}\right)\right|^{-2 s}
$$

(the conditions for positive-definiteness are different for different spaces). Consider the Hilbert space defined by the positive definite kernel $L_{s}(z, u)$. We identify this space with a space of real analytic functions on $G / K$ in the same way as in 2.1. The group $G$ acts on $H_{s}$ by the unitary operators

$$
A_{s}(g) f(z)=f\left(z^{[g]}\right)|\operatorname{det}(a+z c)|^{-2 s}
$$


We call $A_{s}$ a spherical kernel-representation of $G$.

4.8. Nonspherical kernel-representations.

Consider one of the symmetric spaces form $2^{*}, 3^{*}, 5^{*}, 6^{*}, 7^{*}, 9^{*}$, or $10^{*}$ and fix a finite dimensional irreducible representation $\rho$ of the group $G L(n, \mathbb{K})$ or of its universal covering. Assume that the function

$$
L(z, u)=\rho\left(1-z u^{*}\right)
$$

is a matrix valued positive definite kernel. Then we consider the associated Hilbert space $H_{\rho}$ of real-analytic functions $G / K \rightarrow \mathcal{Y}$ and the unitary kernelrepresentation of $G$ in $H_{\rho}$ given by the formula

$$
T_{\rho}(g) f(z)=\rho(a+z c) f\left(z^{[g]}\right)
$$

Consider the cases $1^{*}, 4^{*}, 8^{*}$ and fix a finite-dimensional irreducible representation $\rho=\rho_{1} \otimes \rho_{2}$ of the group $G L(p, \mathbb{K}) \times G L(q, \mathbb{K})$ or of its universal covering. Assume that the function

$$
L_{\rho}(z, u):=\rho_{1}\left(1-z u^{*}\right) \otimes \rho_{2}\left(1-u^{*} z\right)
$$

is a positive definite matrix-valued kernel on $G / K$. Then the group $G$ acts on the associated space of real-analytic functions on $G / K$ by the formula

$$
T_{\rho}(g) f(z)=\left(\rho_{1}(a+z c) \otimes \rho_{2}\left(d-c z^{[g]}\right)\right) f\left(z^{[g]}\right) .
$$

Remark. Our arguments from the Subsections 4.3-4.4 are valid for general kernel-representations.

4.9. Another description of kernel-representations. (see $[\mathrm{O} \varnothing]$ ) For $G=S p(2 n, \mathbb{R}), U(p, q), S O^{*}(2 n)$ a kernel-representation is a tensor product of an irreducible highest weight representation of $G$ and an irreducible lowest weight representation of $G$.

In other cases a kernel-representation of $G$ is a restriction of a highest weight representation of the group $G^{*}$ to the symmetric subgroup $G$ :

$$
\begin{array}{cccccc}
4^{*} . & G=O(p, q) & G^{*}=U(p, q) & 5^{*} . & G=G L(n, \mathbb{R}) & G^{*}=S p(2 n, \mathbb{R}) \\
6^{*} . & G=O(n, \mathbb{C}) & G^{*}=S O^{*}(2 n) & 7^{*} . & G=G L(n, \mathbb{C}) & G^{*}=U(n, n) \\
\text { 8. }^{*} . & G=S p(p, q) & G^{*}=U(2 p, 2 q) & 9^{*} . & G=G L(n, \mathbb{H}) & G^{*}=S O^{*}(2 n) \\
10^{*} . & G=S p(2 n, \mathbb{C}) & G^{*}=S p(4 n, \mathbb{R}) & & &
\end{array}
$$

Remark. The cases $1^{*}-3^{*}$ can be described in the same way. We have $G^{*}=G \times G$ and the embedding $G \rightarrow G^{*}$ given by the formula $g \mapsto\left(g, g^{\theta}\right)$, where $\theta$ is an outer automorphism of $G$.

Remark. There are some additional possibilities related to highest weight representations of $O(p, 2)$ and two exceptional groups (see [OØ]).

4.10. The action of the Olshanskii semigroup. For each matrix ball $G / K$ consider the set of invertible matrices $g=\left(\begin{array}{ll}a & b \\ c & d\end{array}\right)$ such that the 
fractional-linear transformation (16) maps the matrix ball into itself. Obviously $\Gamma$ is a semigroup and the group $G$ is the group of invertible elements of $\Gamma$. The formula (17) defines a representation of the semigroup $\Gamma$. This representation is irreducible and all irreducible representations of $\Gamma$ can be obtained in this way (see [Ols1]). See [Ols1], [Ner3], [Ner4], Appendix A, for the explicit description of the semigroups $\Gamma$. Moreover the kernel-representations extend to representations of some categories (see [Ner3], [Ner4], Appendix A). I don't know any applications of these phenomena to harmonic analysis of kernel-representations of groups.

\subsection{Bibliographical comments.}

a) Let $T$ be a highest weight representation of $G=S p(2 n, \mathbb{R}), S O^{*}(2 n)$, $U(p, q), \ldots$ and $S$ be a lowest weight representation of $G$. Assume that $T, S$ be elements of the Harish-Chandra discrete series. Then $T \otimes S$ is equivalent to a representation of $G$ induced from an irreducible representation of the maximal compact subgroup $K \subset G$ (see [Ber2], [Rep], [Gut]).

b) Spherical kernel-representations for large $s$ are equivalent to canonical representation of $G$ in $L^{2}(G / K)$ (see [OØ]).

c) Discrete spectra associated to the compact orbit in the Shilov boundary were investigated in [NO] for the case $G=O(p, q)$. Analogous results are valid for $G=U(p, q), S p(p, q)$ ([Ols2], [NO], 7.12.). One method of separation of the discrete spectrum is discussed in [Ner1], [NO],7.1-7.8. I think that the restriction operator to the compact orbit doesn't exist for $G \neq U(p, q), S p(p, q), O(p, q)$.

d) The spectra for spherical kernel-representations of $U(2,2)$ were obtained in [ØZ2]. For $1<s<3 / 2$ the spectrum consists of two different pieces. One of the pieces coincides with the spectrum of $L^{2}(U(2,2) /(U(2) \times U(2)))$. The other piece is an integral of nontrivial representations.

Question. It is natural to think (it is not proved) that this piece of the spectrum is associated to the noncompact $U(2,2)$-orbit in the Shilov boundary of $B_{2,2} \times B_{2,2}$. Is it true?

If this is so, then it is the only known case where a spectrum associated to the noncompact orbit can be found. It is natural to think that such spectra exist in various spectral problems (not only for kernel-representations).

e) The Spectrum of spherical kernel-representations of $S U(1,1)$ was obtained in [VGG] and in the case of $U(p, 1)$ it was obtained in [Dij] (the construction of the discrete increments was given in [Ner1]).

f) For a Plancherel formula for tensor product of highest weight and lowest weight (single-valued) representations of $S L(2, \mathbb{R})$, see [Mol5]. Berezin [Ber2] announced a theorem which is almost equivalent to the Plancherel formula for spherical kernel-representation in the case when $s$ is large and the symmetric space is hermitian. Proof is published in [UU].

g) Nonspherical kernel-representations have discrete spectrum which is not associated to the compact orbit in the Shilov boundary. Some possibilities to find it are contained in the following two sections. A way to find the HarishChandra discrete series increments using trace theorems is proposed in [Ner2]. 
h) Let $\rho$ be the same as in 2.10. Let $\rho_{s}(\gamma)=\operatorname{det}^{-s}(\gamma) \rho(\gamma)$. Then the limit of $T_{\rho_{s}}$ as $s \rightarrow \infty$ is the representation of $G$ induced from a finite dimensional representation of $K$ (i.e a representation on the space of sections of a vector bundle on $G / K)$. The theory of such representations is more or less equivalent to Harish-Chandra's theory for $L^{2}(G)$.

e) Deformation quantization, see [Ber2]. As we have seen in $4.2 H_{s}=V_{s} \otimes V_{s}$. Hence we can identify the space $H_{s}$ with the space $V_{s} \rightarrow V_{s}$ of Hilbert-Schmidt operators. The multiplication of Hilbert-Schmidt operators induces an associative operation in $H_{s}$. This operation can be considered as a "deformation quantization", see [Ber3].

\section{Dual pairs}

5.1. Harmonic representation of $S p(2 n, \mathbb{R})$. We recall the definition of the harmonic representation $W_{2 n}$ (= Weil representation = Segal-Shale-Weil representation $=$ Friedrichs-Segal-Berezin-Shale-Weil representation $=$ oscillator representation ) of the group $S p(2 n, \mathbb{R})$ (see $[\mathrm{KV}]$, [Ner4] for a discussion of this representation).

Consider the Hilbert space $F_{n}$ of holomorphic (entire) functions on $\mathbb{C}^{n}$ associated to the reproducing kernel $\exp \left(\sum z_{j} \bar{u}_{j}\right)$ (this is the boson Fock space). Let $\left(\begin{array}{cc}\frac{\Phi}{\Psi} & \Psi\end{array}\right) \in S p(2 n, \mathbb{R})$ (see the realization of $S p(2 n, \mathbb{R})$ described in Subsection 2.4) The harmonic representation is a unitary projective representation of the group $S p(2 n, \mathbb{R})$ in the space $F_{n}$ defined by the Berezin formula (see [Ner4]):

$$
\begin{aligned}
& W\left(\begin{array}{cc}
\Phi & \Psi \\
\Psi & \bar{\Phi}
\end{array}\right) f(z) \\
= & \int_{\mathbb{C}^{\mathbb{N}}} \exp \left\{(z, \bar{u})\left(\begin{array}{cc}
\bar{\Psi} \Phi^{-1} & \Phi^{t-1} \\
\Phi^{-1} & -\Phi^{-1} \Psi
\end{array}\right)\left(\begin{array}{c}
z \\
\bar{u}
\end{array}\right)\right\} f(z) \exp \left(-|z|^{2}\right) d z d \bar{z}(19)
\end{aligned}
$$

5.2. Noncompact dual pairs. Consider the following subgroups in the symplectic group (noncompact Howe dual pairs):

$$
\begin{gathered}
S p(2 k(p+q), \mathbb{R}) \supset S p(2 k, \mathbb{R}) \times O(p, q) \\
S p(2(k+l)(p+q), \mathbb{R}) \supset U(k, l) \times U(p, q) \\
S p(4 k(p+q), \mathbb{R}) \supset S O^{*}(2 k) \times S p(p, q)
\end{gathered}
$$

Remark. Let us describe the first embedding. Consider the space $\mathbb{R}^{2 n}$ equipped with a nondegenerate skew-symmetric bilinear form. Consider the space $\mathbb{R}^{p+q}$ equipped with a symmetric bilinear form with inertia indices $p, q$. The tensor product of symmetric and skew-symmetric forms is a skew-symmetric form in the space $\mathbb{R}^{2 n} \otimes \mathbb{R}^{p+q}$ and we obtain the required embedding of groups. 
Let us restrict the harmonic representation $W_{2 N}$ of the respective large symplectic group to these subgroups and then let us consider the restriction to the subgroups

$$
O(p, q), S p(2 k, \mathbb{R}), U(k, l), U(p, q), S O^{*}(2 k), S p(p, q)
$$

It was proved in [How], [Ada], [Li] that the spectra of these restrictions have discrete increments. This construction is one of the standard ways to obtain singular unitary representations of the groups $U(p, q), O(p, q), S p(p, q)$.

Proposition 5.1. (see [NO]) Each representation of $G=O(p, q), S p(2 k, \mathbb{R})$, $U(k, l), U(p, q), S O^{*}(2 k), S p(p, q)$ which occurs in the spectrum of a dual pair discretely (resp. weakly) occurs in the spectrum of some kernel-representation discretely (resp. weakly).

Proof. This proposition is more or less obvious. Consider for instance the case $S p(2 k, \mathbb{R}) \times O(p, q)$. The restriction of $W_{2 k(p+q)}$ to the subgroup

$$
S p(2 k, \mathbb{R}) \subset S p(2 k, \mathbb{R}) \times O(p, q) \subset S p(2 k(p+q), \mathbb{R})
$$

is equivalent to the representation

$$
W_{2 k}^{\otimes p} \otimes\left(W_{2 k}^{*}\right)^{\otimes q}
$$

The first tensor factor is a direct sum of highest weight representations and the second tensor factor is a direct sum of lowest weight representations. Hence (20) is a direct sum of kernel-representations of the group $\operatorname{Sp}(2 k, \mathbb{R})$.

Consider the subgroup $O(p, q) \subset S p(2 k, \mathbb{R}) \times O(p, q)$. Consider the following subgroups in $S p(2 k(p+q), \mathbb{R})$ :

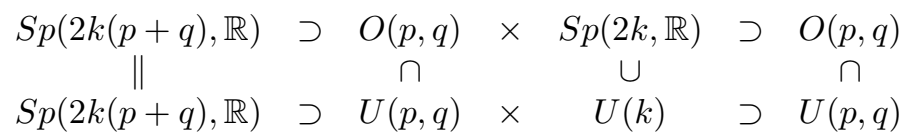

The restriction of $W_{2 k(p+q)}$ to $U(p, q)$ is a direct sum of highest weight representations and hence the restriction of $W_{2 k(p+q)}$ to $O(p, q)$ is a direct sum of kernel-representations.

5.3. Restriction to orbits.

Consider the Cartan domain $C_{N}=S p(2 N, \mathbb{R}) / U(N)$ and the reproducing kernel

$$
K(z, u)=\operatorname{det}^{-1 / 2}\left(1-z u^{*}\right)
$$

on $C_{N}$. Denote by $H$ the associated Hilbert space. The group $S p(2 N, \mathbb{R})$ acts on $H$ by the unitary operators

$$
W_{2 N}^{+}(g)=f\left(z^{[g]}\right) \operatorname{det}^{-1 / 2}(\Phi+z \bar{\Psi})
$$

The representation $W_{2 N}^{+}$is one of two irreducible components of the representation $W_{2 N}$ (the other irreducible component can be realized in some space of 
holomorphic vector-valued functions). Again we have a question about the restrictions of holomorphic functions to $O(p, q) \times S p(2 k, \mathbb{R})$-orbits in the Shilov boundary of $C_{N}$. The orbit structure of the Shilov boundary in this case is very complicated. In any case there exists an orbit

$$
\mathcal{F}=S p(2 k, \mathbb{R}) / U(n) \times O(p, q) / Q,
$$

where $Q$ is stabilizer of a maximal isotropic subspace in the pseudoeuclidean space $\mathbb{R}^{p+q}$. I can show that the restriction operator restricting to the orbit $\mathcal{F}$ exists and this observation gives a way to see a part of the discrete spectrum for the dual pair. It is interesting to calculate this part of the spectrum.

Another question which seems interesting to me: is it possible to obtain by such way some handy realizations of some Harish-Chandra discrete series representations in this way?

\section{The $L^{2}$-space on Stiefel manifolds}

6.1. Stiefel manifolds. The following 10 types of homogeneous spaces $G / Q$ will be called Stiefel manifolds:

$$
\begin{array}{lclc}
1^{\circ} . & O(p, q) / O(p-t, q-s) & 2^{\circ} . & U(p, q) / U(p-t, q-s) \\
3^{\circ} . & S p(p, q) / S p(p-t, q-s) & 4^{\circ} . & S p(2 n, \mathbb{R}) / S p(2(n-t), \mathbb{R}) \\
5^{\circ} . & S p(2 n, \mathbb{C}) / S p(2(n-t), \mathbb{C}) & 6^{\circ} . & O(n, \mathbb{C}) / O(n-t, \mathbb{C}) \\
7^{\circ} . & S O^{*}(2 n) / S O^{*}(2(n-t)) & &
\end{array}
$$

$8^{\circ}-10^{\circ}$. The spaces of all linear embeddings

$$
\mathbb{R}^{n-t} \rightarrow \mathbb{R}^{n} \quad \mathbb{C}^{n-t} \rightarrow \mathbb{C}^{n} \quad \mathbb{H}^{n-t} \rightarrow \mathbb{H}^{n}
$$

In the last 3 cases the group $G$ is $G L(n, \mathbb{R}), G L(n, \mathbb{C}), G L(n, \mathbb{H})$, respectively, and $Q$ is the group of matrices having the form

$$
\left(\begin{array}{cc}
1_{s} & * \\
0 & *
\end{array}\right)
$$

Remark. The Stiefel manifold $S p(2 n, \mathbb{R}) / S p(2(n-t), \mathbb{R})$ is the space of isometric (form preserving) embeddings of the space $\mathbb{R}^{2 t}$ equipped with a nondegenerate skew symmetric bilinear form on the space $\mathbb{R}^{2 n}$ equipped with a nondegenerated skew symmetric bilinear form. The other Stiefel manifolds $1^{\circ}-7^{\circ}$ have an analogous description.

6.2. Additional symmetries.

We consider the homogeneous space $G / Q=S p(2 n, \mathbb{R}) / S p(2(n-t), \mathbb{R})$. Then the group $S p(2 t, \mathbb{R})$ acts in the obvious way on the space of symplectic-isometric 
embeddings $\mathbb{R}^{2 t} \rightarrow \mathbb{R}^{2 n}$ (since it acts on the space $\mathbb{R}^{2 t}$ ). Hence the manifold $S p(2 n, \mathbb{R}) / S p(2(n-t), \mathbb{R})$ is a $S p(2 t, \mathbb{R}) \times S p(2 n, \mathbb{R})$-homogeneous space:

$S p(2 n, \mathbb{R}) / S p(2(n-t), \mathbb{R})=(S p(2 t, \mathbb{R}) \times S p(2 n, \mathbb{R})) /(S p(2 t, \mathbb{R}) \times S p(2(n-t), \mathbb{R}))$

Analogous additional groups of symmetries exist in all the cases $1^{\circ}-10^{\circ}$. These additional symmetries are useful since the spaces $L^{2}(G / Q)$ have $G$-spectrum with infinite multiplicities.

6.3. $L^{2}$-spectrum on Stiefel manifolds. Little is known about the spectral decomposition of $L^{2}(G / Q)$. Nevertheless, this problem is interesting. See [Sch] and [Kob1] for Flensted-Jensen type constructions of discrete spectra in $L^{2}$ on

$$
O(p, q) / O(p-r, q), \quad U(p, q) / U(p-r, q), \quad S p(p, q) / S p(p-r, q)
$$

For the case $r=1$ the Plancherel formula is obtained in [ØZ1]. Some constructions for discrete increments in spectra of

$$
L^{2}(U(p, q) /(U(p-t, q-t) \times U(p) \times U(q))) \subset L^{2}(U(p, q) /(U(p) \times U(q)))
$$

are contained in [RSW].

Remark. The cases $G / Q$, where $G=G L(n, \mathbb{R}), G L(n, \mathbb{C}), G L(n, \mathbb{H})$ are very simple. We can consider two-step unitary induction from the subgroup $Q$ (see (21)) to a parabolic subgroup and from a parabolic to the group $G L(n)$ itself.

Proposition 6.1. Each representation of $G$ which is contained in the spectrum of $L^{2}(G / Q)$ discretely (resp. weakly) is contained in the spectrum of some kernel-representation of $G$ discretely (resp. weakly).

Proof. We use arguments from [How], [NO]. Consider for instance the case $G=\operatorname{Sp}(2 n, \mathbb{R})$. The representation

$$
W_{2 n} \otimes W_{2 n}^{*}
$$

of $S p(2 n, \mathbb{R})$ is equivalent to the representation of $S p(2 n, \mathbb{R})$ in $L^{2}\left(\mathbb{R}^{2 n}\right)$. Hence the representation

$$
\left(W_{2 n} \otimes W_{2 n}^{*}\right)^{\otimes 2 k}=W_{2 n}^{\otimes 2 k} \otimes\left(W_{2 n}^{*}\right)^{\otimes 2 k}
$$

is equivalent to the representation of $S p(2 n, \mathbb{R})$ in $L^{2}$ on the space $M a t_{2 k, 2 n}$ of all $2 k \times 2 n$-matrices. A generic orbit of $S p(2 n, \mathbb{R})$ in $M a t_{2 k, 2 n}$ is a Stiefel manifold $S p(2 n, \mathbb{R}) / S p(2(n-k), \mathbb{R})$.

Remark. For other groups $G$ Proposition 6.1 can be proved using the same arguments. The basic observation is

$$
\left.W_{2 n}\right|_{G L(n, \mathbb{R})} \simeq L^{2}\left(\mathbb{R}^{n}\right)
$$


(cf. the real model of the harmonic representation in [KV]).

6.3. Some pseudo-Riemannian symmetric spaces. In the obvious way we obtain

$$
\begin{array}{ccc}
L^{2}(O(p, q) /(O(p) \times O(p-r, q))) & \subset & L^{2}(O(p, q) / O(p-r, q)) \\
L^{2}(U(p, q) /(U(r) \times U(p-r, q))) & \subset & L^{2}(U(p, q) / U(p-r, q)) \\
L^{2}(S p(p, q) /(S p(r) \times S p(p-r, q))) & \subset & L^{2}(S p(p, q) / S p(p-r, q))
\end{array}
$$

and the spectra of these spaces are contained in the spectra of Stiefel manifolds. I don't know such embeddings of spectra for other pseudo-Riemannian symmetric spaces.

\section{Miscellaneous}

7.1. Molchanov representations. In 1970 Molchanov [Mol2] investigated the most degenerate representations of the groups $O(p, q)$. These representations are obtained by inducing from maximal parabolic subgroups and taking unitary subquotients of these induced representations. Molchanov representations have very simple $K=O(p) \times O(q)$-spectra (spherical harmonics with multiplicities less or equal 1). For this reason Molchanov representations are very simple and handy objects.

These representations are also very singular in different sences: they have small rank in the sence of Howe (on Howe rank see [Li]) and their matrix coeffitients decrease very slowly (on decreasing of matrix elements see $[\mathrm{CHH}]$ ).

Recently these representations attracted interest again, see [HT]. The most degenerate Molchanov representations are now very popular objects, see [BK], $[\mathrm{Kos}],[\mathrm{K} \varnothing]$ (they are minimal representations in the sense of A. Joseph). See in [BK] discussion of minimal representations of $O(p, q)$ and some other series of of simple groups. The most peculiar case is the 'semi-exceptional' group $O(4,4)$, see $[\mathrm{Kos}]$.

I only wanted to mention that Molchanov representations have very interesting spectral properties. Restrictions of Molchanov representations to the subgroup $O(p, q-1)$ were discussed in $[\mathrm{Mol}]$ and $[\mathrm{K} \varnothing]$. The problem is solved for continuous series of Molchanov representations (in this case we have continuous spectrum with discrete increments, see [Mol2]) and for the most degenerate representation (in this case the spectrum is discrete, see $[\mathrm{K} \emptyset]$ ).

Questions. It seems to me that it is a very interesting problem to decompose the tensor product of two Molchanov representations. Another interesting question is 'hunting' for discrete spectra in tensor products of several Molchanov representations (paper [Ner1] contains a false remark on this subject).

Remark. It is possible to show that spectra of tensor products of Molchanov representations contains spectra of dual pairs. I don't know relations between spectra of kernel-representations and spectra of tensor products of Molchanov representations. 
7.2. Representations unitary with respect to indefinite scalar product. There exists a old folk-lor (originated by Ismagilov and Molchanov) on harmonic analysis of represemtations which are unitary in indefinite hermitian scalar products (partially it is published, see [Ism], [Sul]). It is known that these representations often (more often than on definite unitary case) contains discrete increments. It was observed in [Ner1] that this discrete increments usually can be explained by restriction theorems. There arises very much nonsolved problems (for instance it is possible to consider kernel-representations with small or negative parameter $s$ ). Neverless the main (well-known) question now is the following:

Question. Let a representation $\rho$ of a semisimple group $G$ unitary in a indefinite hermitian metric is given. When indefinite scalar product defines by some natural way a topology in space of representation?

The question is very dangerous and sophisticated. On harmonic analysis in handy case of Pontryagin spaces there are several publications, see for instance [Ism], [Sul] (a Pontryagin space is a linear space provided by nondegenerate hermitian form with finite negative inertia index). For some discussion of nonPontraygin case (for highest weight representations) see [HN].

7.3. Harish-Chandra discrete series increments. Tools which are proposed in $[\mathrm{NO}]$ don't give possibilities to search Harish-Chandra discrete series increments to unitary representations. Neverless some possibilities to expose Harish-Chandra discrete series increments with restriction theorems exist (this is the subject of [Ner2]).

7.4. On totaly discrete spectra. In [Ols4], [Kob2] there were discovered large classes or restriction problems which have totaly discrete spectra. Relations between these works are not clear to me, probably they have open nondense intersection. It seems to me that it is interesting to understand are these spectra related to some functional-theretical phenomena or not.

\section{References}

[Ada] Adams, J., Discrete spectrum of reductive dual pair $O(p, q), S p(2 m)$. Inv. math. 74 (1983), 449-475.

[Bar] Bari, N. K., Treatise on trigonometric series. Fizmatgiz (1961) (Russian); English translation:Pergamon Press (1964)

[Ber1] Berezin, F. A., Quantization in complex symmetric spaces, Izv. Akad. Nauk SSSR, Ser. Mat. 39 (1975) (Russian); English translation Math. in USSR Izv. 9 (1975)

[Ber2] -, A connection between the co- and contravariant symbols of operators on classical symmetric spaces. Dokl. Akad. Nauk SSSR 241 (1978), N1(Russian); English translation in Sov. Math. Dokl. 19 (1978), 786-789. 
[Ber3] —, General concept of quantization, Comm. math. phys. 40 (1975), 153174.

[Beu] Beurling, A., Ensembles exceptionels. Acta Math. 72 (1940), 1-13.

[Boy] Boyer, Ch. P., On complementary series of $S O(p, 1)$, J. Math. Phys. 14 (1973), 609-617.

[BK] Brylinski, R., Kostant, B., Minimal representations, geometric quantization and unitarity. Proc. Nac. Acad. Sci. USA 91 (1994), 6026-6029.

[BO] Bunke, U., Olbrich, M., Spectrum of Kleinian manifolds, to appear.

[Cec] Celil T. Lie sphere geometry

[CHH] Cowling M., Haagerup U., Howe R., Almost $L^{2}$-coefficients, J.Reine Angew.Math., 387(1988), 97-110.

[Dij] van Dijk, G., Canonical repesentations, to appear.

[ESh] Egorov, Yu. V., Shubin, M. A., Partial differential equations-1, VINITI, 1987 (Russian); English translation:Encyclopaedia of Mathematical sciences, v.30, Partial differential equations-1.

[EHW] Enright, T.J., Howe, R., Wallach, N., Classification of unitary highest weight modules. in "Representation theory of reductive groups", Progress in Mathematics, Birkhauser, 97-143 (1983).

[Far] Faraut, J., Distributions spheriques sur les espaces hyperboliques. J. Math. Pure Appl. 58 (1979), 369-444.

[Fef] Fefferman, C., Inequalites for strongly singular convolution operators. Acta Math. 124 (1970), 9-36.

[F-J1] Flensted-Jensen, M., Discrete series for semisimple symmetric spaces. Ann. Math. 111 (1980), 253-311.

[F-J2] Flensted-Jensen, M., Analysis on Non-Riemanian symmetric spaces. Regional Conference series in Math. 61, AMS, Providence, 1986.

[GG] Gelfand, I. M., Gindikin, S. G., Complex manifolds whose skeletons are semisimple Lie groups, and analytic discrete series of representations. Funkt. Anal. i Prilozh. 11(1977), N4 (Russian); English translation:Funct. Anal. Appl. 11, 258-265.

[Gin] Gindikin, S. G., Invariant distributions in homogeneous domains. Funkt. Anal. i Prilozh. 9(1975), N1 (Russian); English translation:Funct. Anal. Appl. 9 
[Gut] Gutkin, E., Coefficients of Clebsch-Gordon for the holomorphic discrete series. Lett. Math. Phys. 3 (1979), 185-192.

[H-Ch] Harish-Chandra, Discrete series for semisimple Lie groups. I,II. Acta Math. 113 (1965), 241-318; Acta Math, 116 (1966), 1-111.

[HN] Hilgert J., Neeb K.-H. Unitary highest weight representations in tube domains. Preprint of Mittag-Leffler institute, No.20 1995/96

[How] Howe, R., On some results of Strichartz and of Rallis and Schiffmann. J. Funct. Anal. 32 (1979), 297-303.

[HT] Howe, R., Tan, Homogeneuos functions on light cones: the infinitesimal structure of some degenerate principal series representations. Bull. Amer. Math. Soc. 28 (1993), 1-74.

[Ism] Ismagilov, R. S., On the representations of Lorentz group which are unitary in indefinite metric. Trudy MIEM, 2 (1996), 492-504. (Russian)

[Jac] Jackobsen, H. P., The last place of unitarity for certain highest weight modules. Math. Ann. 256 (1981), 439-447.

[KV] Kashiwara, M., Vergne, M., On the Segal-Shale-Weil representation and harmonic polynomials. Inv. Math. 44 (1978), 1-47

[Kob1] Kobayashi, T., Singular unitary representations and discrete series for indefinite Stiefel manifolds. Mem. AMS 95 (1992).

[Kob2] Kobayashi, T., Discrete decomposability of the restrictions of $A_{\mathfrak{q}}(\lambda)$ with respect to reductive subgroups and its applications. Inv. Math. 117 (1994), 181-205.

[KØ] Kobayashi, T., Ørsted, B., to appear.

[Kos] Kostant, B., The vanishing of scalar curvature and the minimal representation of $O(4,4)$. in "Operator algebras, unitary representations, enveloping algebras and invariant theory", eds. Connes, A., Duflo, M., Joseph, A., Rentschler, R., Birkhäuser, 1990, 85-124.

[Kre] Krein, M. G., Hermitian positive kernels on homogeneous spaces I,II, Ukr. mat. zh. 1 (1949), 4, 64-98; 2 (1950), 1, 10-59 (Russian); English translation:in Translations of Amer. Math. Soc.

[Li] Li, J. S., Singular unitary representations of classical groups. Inv. Math. 97 (1989), 235-257.

[Mol1] Molchanov, V.F., Analogue of the Plancherel formula for hyperboloids. Dokl. Akad. Nauk SSSR 171 (1966) (Russian); English translation:Sov. Math. Dokl 7 (1968), 1382-1385 
[Mol2] —, Representations of pseudoorthogonal group related to cone. Mat. Sbornik 81 (1970), 3, 358-375 (Russian); English translation:Math.USSR Sbornik 10 (1970), 333-347.

[Mol3] —, Restriction of complementary series representations to pseudoorthogonal group of lower dimension. Dokl. Akad. Nauk SSSR 237 (1977), N4 (Russian); English translation:Sov Math. Dokl. 18 (1977), 1493-1497

[Mol4] —, The Plancherel formula for hyperboloids. Trudy MIAN 147 (1980), 65-85 (Russian); English translation:Proc. Steklov Inst. Math. 147 (1981), $63-83$.

[Mol5] —, Tensor products of unitary representations of 3-dimensional Lorentz group. Izv. Akad. Nauk SSSR, ser. Mat. 43 (1979), N4 (Russian); English translation:Math USSR Izv., 15 (1980), 113-143.

[Nai] Naimark, M. A., Decomposition of tensor product of irreducible representations of Lorentz group into irreducible components. III. Trudy Moscovskogo Matem. Obshchestva 10 (1961), 181-216. (Russian)

[NR] Nagel, A., Rudin, W., Local boundary behavior of bounded holomorphic functions. Can. J. Math. 30 (1978), 583-592.

[Ner1] Neretin, Yu. A., Representations of complementary series entering discretely in tensor products of unitary representations. Funkt. Anal. i Prilozh. 20 (1986) N4, 79-80 (Russian); English translation:Funct. Anal. Appl. 20, $68-70$.

[Ner2] —, Restriction of function which is holomorphic in a domain to a curve in boundary, and discrete $S L(2, \mathbb{R})$-spectra. to appear in Izv. Russ. Akad. Nauk.

[Ner3] —, Extensions of representations of classical groups to representations of categories. Algebra i Analiz 3 (1992), N1 (Russian); English translation:StPetersburg Math. J. 3 (1992), 147-169.

[Ner4] - Categories of symmetries and infinite dimensional groups. Oxford Univ. Press (1996)

[Ner5] —, Boundary values of holomorphic functions and discrete spectra in noncommutative harmonic analysis. Proceedings of the summer school "Analysis on homogeneous spaces", Tambov State University, 1997.

[NO] Neretin, Yu. A., Olshanskii, G. I., Boundary values of holomorphic functions, singular unitary representations of groups $O(p, q)$, and their limits as $q \rightarrow \infty$. Zap. Nauchn. Semin. POMIRAN, 223 (1995) 9-91 (Russian); English translation:J. Math. Sci (1996). 
[ÓØ] Ólafsson, G., Ørsted, B., Bargmann transform for symmetric spaces, to appear.

[Ols1] Olshanskii, G. I., Invariant cones in Lie algebra, Lie semigroups, and the holomorphic discrete series. Funkt. Anal. i Prilozh. 14 (1980), N3 (Russian); English translation:Funct. Anal. Appl. 15 (1981).

[Ols2] - Description of highest weight unitary representations for groups $U(p, q)^{\sim}$. Funkt. Anal. i Prilozh. 14 (1980), 3, 32-44 (Russian); English translation: 14 .

[Ols3] —, Singular unitary representations of the groups $O(p, q), U(p, q)$, $S p(p, q)$. (unpublished notes)

[Ols4] -, Unitary irreducible representations of the groups $U(p, q)$ which admit the pass to the limit as $q \rightarrow \infty$. Zap. Nauchn. Semin. LOMI 172 (1989), 114-220. (Russian); English translation:J. Sov. Math. 199.

[OZ1] Ørsted, B., Zhang, G., $L^{2}$-version of Howe correspondence II. J. Math. Pure. Appl. 74 (1995), 165-183.

[OZ2] - Tensor products of analytic continuations of discrete series. Can. J. Math., to appear.

[Pat] Patterson, S. J., The Laplacian operator on a Riemann surface. I,II,III. Comp. Math. 31 (1975), 83-107, Comp. Math. 32 (1976), 71-112, Comp. Math. 33 (1977), 227-259.

[Puk] Pukanszky, L., On the Kronecker products of irreducible representations of the $2 \times 2$ real unimodular group. Trans. Amer. Math. Soc. 100 (1961), $116-152$.

[RSW] Rawnsley, J., Schmid, W., Wolf, J., Singular unitary representations and indefinite harmonic theory, J. Funct. Anal. 51 (1983), 1-114.

[RS] Reed, M., Simon, B., Methods of modern mathematical physics. II. Fourier analysis. Self-adjointness. Acad. Press, 1975.

[Rep] Repka, J., Tensor products of holomorphic discrete series. Can. J. Math. 31 (1979), 836-844.

[RV] Rossi, H., Vergne, M., Analytic continuation of holomorphic discrete series of semisimple Lie group, Acta Math. 136(1976), 1-59.

[Rud] Rudin, W., Function theory in the unit ball in $\mathbb{C}^{n}$. Springer(1980).

[Sch] Schlichtkrull, H., A series of unitary irreducible representations induced from a symmetric subgroup of a semisimple Lie group. Inv. Math. 68 (1982), $497-516$. 
[Str] Strichartz, R. S., Harmonic analysis on hyperboloids. J. Funct. Analysis 12 (1973), 341-383.

[Sul] Sultanov, Sh. Sh. On tensor products of representations of the group $S L_{2}(\mathbb{R})$. Funkt. Anal. i Prilozh. 11 (1977), N 4, 90-92 (Russian); English translation:Funct. Anal. Appl. 11.

[Tsu] Tsuchikawa, The Plancherel transform on $S L_{2}(k)$ and its applications to tensor products of irreducible representations. J. Kyoto Univ. 22 (1982), $369-473$.

[UU] Unterberger, A., Upmeier, H. The Berezin transform and invariant differential operators. Comm. Math. Phys., 164(1994), 563-597.

[VGG] Vershik, A.M., Gelfand, I.M., Graev, M.I., Representations of $S L(2, R)$ where $R$ is function ring. Uspekhi Mat. Nauk 28 (1973), 5, 83-128 (Russian); English translation:Russian Math. Survey, 28.

[Vla] Vladimirov, V. S., On constructions of envelopes of holomorphy for special domains and its applications. Trudy MIAN (Proceedings of Steklov Institute), 60 (1961), 101-144. (Russian)

[VS] Vladimirov, V.S., Sergeev, A.G., Complex analysis in future tube. Complex analysis: several variables-2. Moscow:VINITI, 1985, 191-260 (Russian); English translation:in Encyclopaedia of mathematical sciences, v.8, "Complex analysis. Several variables-2", Springer.

[Wal] Wallach, N., The analytic continuation of discrete series I,II., Trans. Amer. Math. Soc. 251 (1979), 1-17, 19-37. 